IdeAs

Idées d'Amériques

4 | 2013

Crises et effets de crise dans les Amériques

\title{
« Paying your Visa with your MasterCard »: La spirale infernale des cartes de crédit aux États-Unis
}

"Paying your Visa with your MasterCard " : the Credit Card Trap in the United States

"Paying your Visa with your MasterCard : La espiral infernal de las tarjetas de crédito en los Estados Unidos

\section{Marie-Christine Pauwels}

\section{OpenEdition}

\section{Journals}

Édition électronique

URL : https://journals.openedition.org/ideas/736

DOI : 10.4000/ideas.736

ISSN : 1950-5701

Éditeur

Institut des Amériques

Référence électronique

Marie-Christine Pauwels, « «Paying your Visa with your MasterCard »: La spirale infernale des cartes de crédit aux États-Unis », IdeAs [En ligne], 4 | 2013, mis en ligne le 15 octobre 2013, consulté le 19 octobre 2022. URL : http://journals.openedition.org/ideas/736 ; DOI : https://doi.org/10.4000/ideas 736

Ce document a été généré automatiquement le 19 octobre 2022

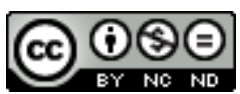

Creative Commons - Attribution - Pas d'Utilisation Commerciale - Pas de Modification 4.0 International - CC BY-NC-ND 4.0

https://creativecommons.org/licenses/by-nc-nd/4.0/ 


\title{
« Paying your Visa with your MasterCard $»^{1}$ : La spirale infernale des cartes de crédit aux États-Unis
}

\author{
"Paying your Visa with your MasterCard": the Credit Card Trap in the United \\ States \\ "Paying your Visa with your MasterCard : La espiral infernal de las tarjetas de \\ crédito en los Estados Unidos
}

Marie-Christine Pauwels

1 Cet article se propose d'illustrer le phénomène du surendettement aux États-Unis à travers le phénomène des cartes de crédit. Après une première partie consacrée aux raisons historiques du boom du crédit à la consommation en général et à la démultiplication des cartes de crédit en particulier, nous décortiquerons certaines des pratiques et des stratégies utilisées par les banques et sociétés de crédit pour élargir leur base de clients et pénétrer de nouveaux marchés. Une dernière partie s'interrogera sur les dérives de cette sur-utilisation des cartes de crédit, notamment les répercussions dramatiques sur les emprunteurs les plus vulnérables dans le contexte de la crise financière qui frappe le pays depuis 2008-2009.

\section{Dérèglementation financière et révolution technologique}

2 L'essor du crédit à la consommation en général et des cartes de crédit en particulier remonte à une trentaine d'années, avec une série de décisions qui ont libéralisé le secteur bancaire.

- 1978 : l'arrêt de la Cour suprême Marquette National Bank of Minneapolis v. First National Bank of Omaha libère les plafonds sur les taux d'intérêt. La fixation du taux d'intérêt d'un prêt dépend désormais des règles en vigueur dans l'État où est implanté la banque ou l'organisme de crédit, et non plus de celles de l'État où réside l'emprunteur. Conséquence immédiate de 
cette décision, le Delaware et le Dakota du sud, deux États où les taux d'intérêt sont peu réglementés, voient affluer les sociétés financières qui y décentralisent leurs opérations de crédit. En 1981, Citibank déménage son pôle cartes de crédit de New York dans le Dakota du sud.

- 1996 : dans un nouvel arrêt, Smiley v. Citibank, la Cour suprême libère les taux de pénalités et les frais divers sur les cartes de crédit. Celles-ci, précédemment de l'ordre de 5-10 dollars, connaissent une véritable explosion (avoisinant désormais les 50 dollars).

- 1999 : la loi sur la modernisation des services financiers (Financial Services Modernization Act FSMA) abroge le Glass-Steagall Act de 1933 qui instaurait une séparation entre banques commerciales et banques de dépôt, ainsi que le McFadden Act de 1927 qui restreignait les transactions bancaires inter-États. C'est le début d'un processus de consolidation du secteur et de la naissance des énormes conglomérats bancaires tel Citigroup qui voit le jour en 1998.

- 2000 : pour éviter une récession, la Réserve fédérale entame une baisse de son taux directeur (de 9,5\% en 2000 à $4 \%$ en 2003) et donc le prix du crédit. Ménages et particuliers n'hésitent plus à s'endetter, et la consommation s'envole.

3 Autre facteur d'essor, l'apparition sur le marché de logiciels de plus en plus performants et capables d'analyser avec minutie les risques potentiels que pose chaque emprunteur. Une micro-segmentation des consommateurs selon leur profil d'endettement voit ainsi le jour. Différents paramètres sont mesurés par un indice appelé FICO - du nom de la société ayant mis au point ce procédé, la Fair Isaac Corporation - et permettent aux banques d'offrir des prêts véritablement sur mesure, personnalisés selon le profil de chacun. Compris entre 300 et 850 , cet indice est utilisé par tous les organismes de prêt. Plus il est élevé, meilleures sont les conditions du prêt. Un bon score se situe au-dessus de 680.

Ces outils de customisation de plus en plus sophistiqués ont pour effet de démocratiser le crédit à la consommation et le marché des cartes de crédit s'emballe. Avant 1990, seuls les consommateurs les plus aisés se voyaient proposer une carte de crédit, à un taux d'intérêt fixe (généralement aux alentours de $20 \%$ ). Désormais, elles deviennent accessibles aux bas revenus aussi.

5 En 2005, le Président de la Réserve fédérale, Alan Greenspan, souligne l'aspect vertueux de cet élargissement du crédit aux moins fortunés. Il y voit une forme de progrès social, la possibilité pour les ménages modestes, grâce à l'endettement, d'accéder à la propriété et à la société de consommation. L'accès au crédit devient le sésame qui leur permettra de concrétiser le rêve américain : « il est incontestable que l'innovation et la déréglementation ont permis à pratiquement tous les ménages, quels que soient leurs revenus, d'avoir accès au crédit. Grâce à cela, de nombreuses familles ont pu accéder à la propriété, répondre aux situations de crise et s'offrir de nombreux biens et services $»^{2}$.

6 En raison de cette expansion très rapide du marché, chaque Américain possède désormais en moyenne dix à douze cartes de paiement, essentiellement fournies par les quatre grands réseaux que sont Visa, MasterCard, American Express, et Discover. Il convient de préciser que toutes ces cartes ne sont pas des cartes de crédit. Les cartes de débit qui sont, elles, directement reliées à un compte bancaire - l'équivalent français de nos cartes de crédit - ne ne sont pas celles qui nous intéressent ici. Ce sont les cartes de crédit, qui peuvent être délivrées par divers organismes, banques, sociétés de crédit, grandes enseignes de la distribution, etc., et qui offrent une ligne de crédit renouvelable, le fameux revolving, qui font l'objet de cette étude. 
7 Car le crédit revolving donne naissance à deux types d'utilisateurs, l'un appelé convenience user ( $45 \%$ des utilisateurs), toujours solvable, qui rembourse scrupuleusement ses emprunts tous les mois, n'a jamais de retard de paiement et maîtrise bien le système, et l'utilisateur imprudent, appelé revolver, qui se laisse déborder, gère mal son budget, est incapable de faire face à ses échéances mensuelles et se retrouve pris au piège. Et contrairement à ce que clament les banques et les organismes de crédit dont le discours met en avant la responsabilité personnelle, louant la gestion de bon père de famille des convenience users, ce sont les revolvers qui sont les plus rentables, compte tenu des multiples frais, pénalités de retard et envol des taux d'intérêt qu'ils subissent au moindre problème. La réalité inavouable est que ces clients mauvais payeurs sont devenus les véritables vaches à lait du système.

8 Pourquoi cette dérive ? De la même manière que la titrisation sur le marché boursier permet de diluer les risques en revendant aux investisseurs des packages de titres où créances solides et créances douteuses sont mises dans le même sac, il y a eu sur le marché du crédit à la consommation un phénomène identique qui a entraîné une forme de déresponsabilisation des acteurs du système.

9 Pour illustrer ce phénomène, prenons l'exemple d'un particulier ayant contracté un crédit de 100 dollars auprès d'un organisme de prêt: ce dernier recombine alors ce crédit avec d'autres et le vend 115 dollars sous forme de package of credit card securities à des investisseurs anticipant des retards de remboursements sur ces crédits et donc des pénalités accrues pour l'emprunteur. L'organisme ayant consenti le prêt gagne ainsi de l'argent grâce aux intérêts de remboursement en spéculant sur les mauvais payeurs. De la sorte, il lui importe moins d'être scrupuleusement remboursé chaque mois que d'avoir des clients qui accumulent les retards de paiement afin de pouvoir déclencher la spirale de pénalités et de frais divers.

10 En résumé, un bon client est un emprunteur qui ne rembourse jamais son prêt. Le système engendre ses propres effets pernicieux, et l'aléa moral, principe qui stipule qu'un agent économique, isolé d'un risque, se comportera de manière moins responsable que s'il devait en subir directement les conséquences, s'en trouve par conséquent accru. La moitié des profits du secteur proviendrait des pénalités et des frais prélevés sur ces mauvais payeurs (Surowiecki, 2009).

\section{Un marketing agressif}

11 Compte tenu de cette logique, il n'est guère surprenant de constater l'agressivité des campagnes de promotion menées par le secteur afin d'élargir sa base de clients. C'est un véritable matraquage d'offres de souscription que subissent les Américains, plusieurs millions de courriers atterrissant chaque semaine dans leurs boîtes aux lettres. Le nombre de publicités envoyées à domicile a quasiment triplé en dix ans. Toutes proposent des conditions plus alléchantes les unes que les autres, notamment un taux d'intérêt initial de $0 \%$ pendant 6 mois (appelé teaser rate), ce qui est devenu la norme pour ce genre de sollicitation. Sont tout particulièrement visées certaines catégories de population plus fragiles, notamment les ménages modestes, les jeunes et les étudiants. Une étude de la Fed datant de 2005 et portant sur les années 1989 à 2001 fait état de l'augmentation phénoménale du nombre de cartes chez les ménages les moins fortunés en l'espace d'une douzaine d'années : + $50 \%$ (de $29 \%$ à $43 \%$ ), contre + $10 \%$ (de $70 \%$ à $76 \%$ ) au sein de la population globale (Johnson, $2005: 475$ ). 
Également ciblés, les pré-adolescents ou tweens (en 2004, la société Legend Credit lance une gamme de cartes à l'effigie de la mascotte Hello Kitty), et les étudiants, marché qui va connaître une augmentation spectaculaire, comme en témoignent les chiffres cidessous :

-à la fin des années 1980, moins de $40 \%$ des étudiants ont une carte de crédit contre $84 \%$ en 2009;

- un étudiant de $1^{\text {ère }}$ année reçoit en moyenne 8 offres de cartes de crédit la première semaine de cours;

- Les deux-tiers des étudiants sont endettés, contre la moitié dans les années 1990 ;

- le taux d'endettement moyen par étudiant en fin d'études dans les années 2000 est supérieur à 3000 dollars, auquel se rajoutent quelque 20000 dollars de prêt étudiant (Sallie Mae : 2009).

Cette évolution est d'autant plus grave qu'un historique d'endettement poursuit le jeune bien après ses années d'études. Un mauvais indice FICO peut être utilisé pour justifier un refus d'embauche, des taux d'intérêt plus élevés lors d'un emprunt futur, des mensualités plus lourdes, etc. Se produit alors un phénomène de blacklisting insidieux dont la plupart de ces jeunes gens n'ont pas conscience.

Autre dérive à noter aujourd'hui, l'avancée du secteur dans le domaine de l'assurancesanté, avec l'apparition de nouvelles cartes de crédit à l'usage exclusif du paiement des soins médicaux. Certains hôpitaux, voulant être sûrs de rentrer dans leurs frais, demandent désormais aux patients de leur fournir une carte de crédit avant toute prise en charge (Zeldin et Rukavina, 2007 ; Rowland, 2007 : A1).

\section{Des pratiques contestables}

Les organismes de cartes de crédit font depuis plusieurs années l'objet d'une surveillance accrue de la part des pouvoirs publics et des associations de consommateurs qui leur reprochent, outre de viser des publics vulnérables, de multiplier les pratiques contestables visant à berner l'utilisateur, ou tout au moins à le sous-informer de manière plus ou moins volontaire.

Les contrats de souscription sont en effet compliqués, truffés de clauses peu explicites, voire incompréhensibles, et ce qui n'arrange rien, réajustables a posteriori par l'organisme de prêt. Au consommateur de s'y retrouver entre le taux d'intérêt initial très attractif, en général de $0 \%$ pendant les premiers six mois, et les autres taux qu'il faut aussi prendre en compte : l'un rentre en vigueur au terme des six premiers mois, un autre est appliqué pour les transferts, un troisième pour les avances de liquide, etc. Difficile également de faire la part des choses entre tous les frais divers (expédition, assurance, frais de dossier...) ; sans compter les pénalités de retard, les dates limites de remboursement qui peuvent tomber le week-end ou bien changer d'un mois sur l'autre... Dans cette liste sans fin, les deux pratiques les plus contestables sont la facturation sur deux cycles (double-cycle billing, l'intérêt étant appliqué de manière rétroactive sur une dette déjà remboursée) et le défaut universel (universal default, un seul retard de paiement sur une carte de crédit pouvant entraîner l'augmentation des taux d'intérêt sur tous les autres prêts contractés par l'emprunteur). Cette pratique a été interdite en 2010. 
17 Dans un documentaire de la chaîne PBS datant de 2004, Elizabeth Warren, professeur de droit des contrats à Harvard et l'une des figures de proue de la lutte contre les pratiques abusives du secteur, s'insurge contre la profession qu'elle accuse de duperie : « ces gars-là n'ont rien trouvé de mieux pour écraser leurs concurrents que de faire des pubs alléchantes, de vous appâter avec un taux d'intérêt initial très bas, et de s'entourer d'une équipe d'experts pour vous piéger avec des contrats incompréhensibles $»^{3}$.

18 Autre motif de mécontentement, le flou qui entoure à l'époque les instances régulatrices, personne ne sachant véritablement vers qui se tourner en cas de litige. Selon le type de charte sous laquelle la banque opère, elle est régulée par l'État fédéral ou au niveau de chaque État. Et rien qu'au niveau fédéral, cinq agences différentes contrôlent chacune une partie du système : la Fed, l'OCC (Office of the Comptroller of the Currency), l'OTS (Office of Thrift Supervision), la FDIC (Federal Deposit Insurance Corporation) et la FTC (Federal Trade Commission).

19 Face à une telle complexité, difficile pour le consommateur de s'y retrouver. En outre, ajoutons que la plupart des Américains n'ont qu'une connaissance très approximative des mécanismes financiers. L'ampleur de la crise s'explique aussi tout simplement par la phénoménale asymétrie d'informations qui existe entre une industrie de plus en plus sophistiquée qui propose des produits financiers d'une haute complexité à des individus souvent mal renseignés ou ignorant les principes de base de la finance. Nombreux sont les emprunteurs à juger un crédit abordable si le taux d'intérêt initial est peu élevé et les mensualités suffisamment basses pour rentrer dans leur budget mensuel. Le coût total du crédit sur le long terme, paramètre pourtant le plus essentiel, est perçu comme secondaire.

Selon une étude menée par le Government Accountability Office (GAO) en 2006, 50 \% des utilisateurs de cartes de crédit reconnaissaient ne pas comprendre les termes du contrat qui les engage (GAO report, 2006). Dans un sondage effectué l'année suivante, plus d'un tiers des adultes interrogés ( $37 \%$ ) avouaient ignorer leur score FICO, près des deux-tiers (64\%, soit 144 millions d'individus) n'avaient pas demandé à consulter leur historique de crédit pour l'année en cours (cette proportion atteignant même $72 \%$ chez les Hispaniques), et les trois-quarts admettaient ne jamais lire leur contrat ${ }^{4}$.

\section{Le surendettement comme mode de vie}

21 Cela dit, il convient de nuancer cette image du consommateur, simple victime passive d'une industrie prédatrice, car dans cette spirale du surendettement, les changements socio-culturels et l'évolution considérable des mentalités vis-à-vis du crédit ont également joué un rôle.

Depuis trente ans, le crédit s'est démocratisé et banalisé. Jusqu'à la crise récente qui a conduit les Américains à s'interroger en profondeur sur les dérives du système, vivre à crédit n'était plus comme par le passé teinté d'opprobre ; c'était devenu au contraire la marque d'un comportement rationnel et astucieux, un moyen malin pour boucler son budget. Dans un complet renversement de tendance, refuser de vivre à crédit était stigmatisé comme un comportement vieux jeu, obsolète et dépassé.

23 En parallèle au phénomène de surconsommation qui caractérise les États-Unis contemporains s'est produit un phénomène de «ringardisation" de l'épargne, les 
écueils d'un endettement mal maîtrisé étant de plus en plus souvent camouflés sous un discours prônant la gratification du plaisir immédiat. Cette morale hédoniste est habilement exploitée par les annonceurs qui font appel à des ressorts psychologiques bien rodés, fondés notamment sur la déculpabilisation de l'utilisateur. Les campagnes de pub MasterCard, par exemple, insistent sur le fait que les cartes de crédit sont irremplaçables parce qu'elles simplifient la vie, libèrent des contingences immédiates et permettent de profiter de moments précieux en famille : «l'argent ne peut pas tout acheter. Pour tout le reste, il y a MasterCard $»^{5}$. Les cartes de crédit permettent également de flatter la vanité de certains qui se voient proposer des produits haut de gamme leur ouvrant les portes des clubs sélects et leur donnant l'illusion de faire partie des happy few : Visa propose ainsi la carte Premier, la Gold, la Platinum, et sa dernièrenée, la Black Card, fabriquée non en vulgaire plastique mais en carbone, et réservée au $1 \%$ les plus fortunés; chez MasterCard ce sont les cartes Diamond ou Titanium, chez American Express la carte Centurion, etc. On assiste ainsi à une véritable surenchère de cartes prestiges, toutes plus élitistes les unes que les autres.

Autre levier psychologique, celui des récompenses: une carte de crédit, c'est l'assurance de pouvoir gagner des miles, bénéficier de pleins d'essence gratuits, de chèques cadeaux, de réductions diverses et variées, et d'un service 5 étoiles dans tous les établissements partenaires. L'imagination des annonceurs est dans ce domaine sans limites.

Enfin, pour faire tomber les dernières réticences, la profession a également mis sur le marché des cartes caritatives appelées affinity cards, qui garantissent au souscripteur le reversement d'un pourcentage des frais prélevés (en général, moins de $1 \%$ ) à la cause de son choix. Outre la récupération d'image pour la banque qui, en surfant sur la vague éthique s'affiche comme un acteur socialement responsable, l'utilisateur peut ainsi faire œuvre utile et se donner bonne conscience en sauvant les dauphins, les baleines ou le littoral en danger, en aidant les vétérans, les handicapés, les enfants dans le besoin, etc.

Faciles à obtenir, faciles à utiliser, les cartes de crédit sont devenues indispensables. Impossible aujourd'hui sans carte de crédit de réserver une chambre d'hôtel, de louer une voiture, ou d'effectuer un achat en ligne. Une proportion croissante d'Américains a pris l'habitude de les utiliser pour les dépenses courantes, y compris les plus modestes comme un café chez Starbucks ou une part de pizza. Que ce soit pour faire le plein d'essence, les courses au supermarché, payer sa facture de téléphone, d'électricité, son loyer, et même ses impôts et ses frais médicaux, l'Amérique voue un véritable culte au rectangle de plastique.

\section{«Paying your Visa with your MasterCard »}

Pour certains, grisés par l'illusion de l'argent facile, les cartes de crédit ont entraîné une perte du sens des réalités. L'aveuglement a engendré l'imprudence et cette spirale du crédit est devenue incontrôlable.

En 2009, trois consommateurs sur quatre, l'équivalent de 90 millions de ménages, étaient endettés auprès d'un organisme de carte de crédit, et le taux d'endettement moyen par foyer dépassait les 10500 dollars (Nilson report, 2009). 
29 Le processus est toujours le même : cédant aux sirènes du crédit revolving, de nombreux Américains ont souscrit à quantité d'offres de cartes, enclenchant ainsi une spirale infernale et se retrouvant pris au piège. Pire, l'habitude a été prise de jongler (shuffling) entre les différentes cartes au fur et à mesure que les plafonds autorisés sont atteints. Ainsi, lorsque la ligne de crédit d'une première carte est épuisée (maxed out), le solde est basculé sur une deuxième, puis éventuellement une troisième, et ainsi de suite. Cette pratique très risquée, volontiers tournée en dérision - «paying your Visa with your MasterCard» - ne se limite d'ailleurs pas au seul secteur des cartes de crédit, mais s'est étendue par contagion au secteur des prêts hypothécaires, donnant naissance au phénomène de refinancement de crédit. Ainsi, un quart des propriétaires ayant contracté un prêt bancaire pour acheter leur maison se sont laissés persuader de contracter un deuxième emprunt, une stratégie conseillée par les experts financiers au moment où l'immobilier flambe (le prix des maisons double entre 2001 et 2005 par exemple). Il est présenté comme très avantageux à l'époque de financer son crédit à la consommation (dont le taux d'intérêt avoisine en général $18 \%$ ) grâce à ce nouvel emprunt hypothécaire au taux d'intérêt bien plus faible (de l'ordre de $6 \%$ ). Gager son toit devient pratique courante, comme en témoigne cette publicité de la banque Citigroup en 2003 : «Votre maison vous a demandé un gros investissement. Vous ne croyez pas qu'il est temps de la mettre à contribution ? $"^{6}$

Cette interconnexion entre crédit immobilier et crédit à la consommation, qui rappelle une autre pratique à risque, celle des fameux prêts NINJA (No Income, No Job, (and) no Assets ${ }^{7}$ ), ces prêts subprime consentis à des emprunteurs sans revenus, sans emploi et sans capital, a ainsi contribué à fragiliser un peu plus tout l'édifice.

31 Depuis le déclenchement de la crise, les faillites personnelles ont explosé, et ce, malgré le Bankruptcy Abuse Prevention and Consumer Protection Act de 2005 qui cherchait à limiter les abus en rendant la procédure plus onéreuse et plus restrictive. En 2009, 1,6 million d'Américains ont fait faillite, soit le double de 2007. Dans $60 \%$ des cas, ces faillites sont liées à un problème de santé (Simkovic, 2009 : 1-26).

On voit bien qu'au-delà d'un problème d'imprudence personnelle, c'est tout un système qui marche sur la tête. Lors de l'éclatement de la bulle immobilière, l'écart entre revenus et dépenses s'est tellement creusé que les cartes de crédit sont devenues le seul moyen pour les plus bas revenus de pallier les salaires qui stagnent, le chômage, la faiblesse des allocations, les revenus immobiliers qui dégringolent, les frais de santé et d'éducation en hausse, etc.

33 Car le surendettement des ménages est autant dû à une frénésie de surconsommation et à l'attrait de l'argent facile qu'à une dégradation des conditions de vie et à un déclassement des plus vulnérables. Pour de nombreux Américains de la classe moyenne, pris en tenaille entre revenus à la baisse et dépenses à la hausse, le crédit revolving est souvent, bien malgré eux, devenu le dernier recours, une sorte de précaire filet de sécurité. Pour les catégories socio-professionnelles les moins aisées, vivre à crédit et jongler entre les différentes cartes de crédit n'est même plus une question de choix, c'est devenu une nécessité. Parmi les nombreuses études faisant état de cette fuite en avant, trois rapports effectués entre 2007 et 2012 par les chercheurs du think tank Dēmos de New York, ont constaté que le pourcentage de ménages à bas revenus ayant recours aux cartes de crédit pour leurs dépenses quotidiennes était passé de $32 \%$ à 40 \% (Drault et Wheary, 2007 ; Traub et Ruetschlin, 2009 ; Draut et Garcia, 2012). 

Congrès, la Credit Card Accountability, Responsibility, and Disclosure Act (CARD), aussi appelée the Credit Card Bill of Rights, signée par le président Obama en mai 2009. L'objectif de cette loi est de moraliser le secteur bancaire tout entier en éliminant les abus les plus criants. Elle inclut notamment un meilleur encadrement des hausses des taux d'intérêt, limite l'offre de crédit en direction des étudiants en interdisant la souscription de jeunes de moins de 21 ans sans contrôle parental ou vérification de leur solvabilité afin d'éviter les dérapages. Autres mesures phares : l'obligation d'accorder un délai de 45 jours à l'emprunteur avant toute augmentation des taux d'intérêt, l'interdiction de la pratique du double-cycle billing et de la clause du défaut universel ainsi que les pénalités rétroactives.

Et en 2010, la loi de réforme financière Dodd-Frank crée le Consumer Financial Protection Bureau (CFPB), un nouvel organisme chargé de coordonner l'action des différentes instances régulatrices et d'assurer un meilleur contrôle sur l'ensemble du secteur financier afin de mieux informer le consommateur et le protéger contre les pratiques abusives.

La réponse de la profession ne s'est pas fait attendre:le législateur ayant laissé quelques mois aux banques pour se mettre en conformité avec ces nouvelles dispositions, ces dernières se sont empressées d'augmenter leurs taux d'intérêt et leurs frais divers, de se débarrasser des clients les moins solvables ou les plus risqués, et ont mené des campagnes de lobbying intense pour neutraliser les mesures les plus drastiques. Elles ont ainsi obtenu qu'aucun plafonnement des taux d'intérêt ne leur soit imposé par exemple (Lieber et Martin, 2009 : A1).

Mais les choses se sont malgré tout améliorées. Selon la toute dernière étude que Dēmos a consacré à ce sujet en 2012, le nombre de ménages assujetis à des pénalités de retard a considérablement diminué depuis 2008, passant de $50 \%$ à $28 \%$. Et ils ne sont plus que $29 \%$ contre $53 \%$ en 2008 à se voir infliger des hausses de taux d'intérêt en cas de retards de paiement. L'endettement moyen des ménages auprès des organismes de cartes de crédit a également diminué, 7145 dollars contre 9887 dollars en 2008. Le tableau ci-dessous permet de mieux se rendre compte de l'amélioration de la situation pour chaque catégorie de consommateur (Traub et Ruetschlin, 2012:6).

Tableau 1. Endettement auprès d'organismes de cartes de crédit

\begin{tabular}{|l|l|l|l|}
\hline & 2008 & 2012 & $\%$ changement \\
\hline Total & $\$ 9887$ & $\$ 7145$ & $-27,7 \%$ \\
\hline Age & & & \\
\hline $18-24$ & $\$ 3498$ & $\$ 2982$ & $-14,7 \%$ \\
\hline $25-34$ & $\$ 10407$ & $\$ 5156$ & $-50,5 \%$ \\
\hline $35-44$ & $\$ 10141$ & $\$ 6156$ & $-39,3 \%$ \\
\hline $45-54$ & $\$ 10154$ & $\$ 8408$ & $-17,2 \%$ \\
\hline
\end{tabular}




\begin{tabular}{|l|l|l|l|}
\hline $55-64$ & $\$ 10013$ & $\$ 8228$ & $-17,8 \%$ \\
\hline 65 et plus & $\$ 9823$ & $\$ 9283$ & $-5,5 \%$ \\
\hline Ethnicité & & & \\
\hline Noirs & $\$ 6970$ & $\$ 5784$ & $-17,0 \%$ \\
\hline Hispaniques & $\$ 9049$ & $\$ 6066$ & $-33,0 \%$ \\
\hline Blancs, non-Hispaniques & $\$ 10358$ & $\$ 7315$ & $-29,4 \%$ \\
\hline Revenus & & & \\
\hline$<\$ 35000$ & $\$ 7763$ & $\$ 5405$ & $-30,4 \%$ \\
\hline$\$ 35-49000$ & $\$ 10206$ & $\$ 6736$ & $-34,0 \%$ \\
\hline$\$ 50-74999$ & $\$ 11528$ & $\$ 8916$ & $-22,7 \%$ \\
\hline$\$ 75000<$ & $\$ 11896$ & $\$ 9235$ & $-22,4 \%$ \\
\hline
\end{tabular}

\section{Conclusion}

À l'illusion du crédit facile et de la prospérité pour tous grâce à la magie des cartes de crédit s'est substituée une réalité moins rose, une réalité qui a fait apparaître au grand jour le creusement des inégalités face à l'endettement en général et aux cartes de crédit en particulier. C'est bel et bien un système à deux vitesses qui s'est installé, entre les catégories aisées qui ont pu bénéficier de taux d'emprunt préférentiels et les plus vulnérables qui se sont vues proposer des taux punitifs. Les banques se défendent en prétextant un plus fort niveau de risque, arguments que réfutent les experts comme Robert Manning, économiste au Rochester Institute of Technology et l'un des plus grands spécialistes des abus du secteur des cartes de crédit: «(...) l'argument selon lequel l'équilibre se fait entre d'un côté le 'bon' débiteur à qui la banque va proposer des taux d'intérêt avantageux en récompense de sa gestion prudente et de l'autre, le 'mauvais', pénalisé par des taux élevés parce qu'il a souscrit un crédit revolving est de plus en plus souvent dénoncé comme moralement inacceptable $»^{8}$.

En d'autres termes, l'argument libéral classique qui oppose l'utilisateur prudent et discipliné à l'irresponsable qui flambe et cède aux sirènes de l'argent facile, et qui nous renvoie à l'un des grands mythes de la société américaine, à savoir l'opposition entre l'individu méritant et responsable et le parasite qui vit aux crochets de la société, s'avère totalement spécieux. Les cartes de crédit sont dans l'ensemble des produits extrêmement coûteux pour le consommateur et offrent un nouvel éclairage sur le phénomène de l'accroissement des inégalités aux États-Unis entre ceux qui maîtrisent ces produits complexes et les autres qui en sont victimes. 


\section{BIBLIOGRAPHIE}

CreditCards.com, Enquête de Juin 2007. Disponible en ligne : http://www.creditcards.com/creditcard-news/credit-card-industry-facts-personal-debt-statistics-1276.php.

Draut, Tamara, et Jennifer Wheary, « Who Pays ? The Winners and Losers of Credit Card Deregulation », in Dēmos Report, $1^{\mathrm{er}}$ août, 2007. Disponible en ligne : http://www.demos.org/sites/ default/files/publications/whopays_Demos.pdf.

Draut, Tamara, et Jose Garcia, " The Plastic Safety Net : How Households are Coping in a Fragile Economy ", in Dēmos Report, 28 juillet 2009. Disponible en ligne : http://www.demos.org/sites/ default/files/publications/PlasticSafetyNet_Demos.pdf.

Frontline \& NYT, « Secret History of the Credit Card », A Frontline \& NYT Documentary, 23 novembre 2004. Disponible en ligne $:$ http://www.pbs.org/wgbh/pages/frontline/shows/credit/ view/.

Greenspan, Alan, Consumer Finance, remarks presented at the Federal Reserve System's Fourth Annual Community Affairs Research Conference, Washington, D.C., 8 avril 2005.

Johnson, Kathleen, « Recent Developments in the Credit Card Market and the Financial Obligations Ratio ", in Fed. Reserve Bulletin, automne 2005, p. 475. Disponible en ligne : http:// www.federalreserve.gov/pubs/bulletin/2005/autumn05_lead.pdf.

Lieber, Ron, et Andrew Martin, « Overspending on Debit Cards is a Boon for Banks ", in New York Times, 9 septembre 2009, p. A1.

Manning, Robert, Credit Card Nation, The Consequences of America's Addiction to Credit, New York, Basic Books, 2000.

National Foundation for Credit Counseling, 2009 Financial Literacy Survey, avril 2009.

Nilson Report, n 924, avril 2009.

Rowland, Christopher, « Patients Piling Medical Costs on Credit Cards », in The Boston Globe, 22 janvier 2007, p. A1. Disponible en ligne : http://www.boston.com/business/globe/articles/ 2007/01/22/patients_piling_medical_costs_on_credit_cards/.

Sallie Mae's National Study of Usage Rates and Trends 2009, « How Undergraduate Students Use Credit Cards ", Rapport, Avril 2009. Disponible en ligne : http://inpathways.net/ SLMCreditCardUsageStudy41309FINAL2.pdf.

Simkovic, Michael, « The Effect of the 2005 Bankruptcy Reforms on Credit Card Industry Profits and Prices ", in American Bankruptcy Law Journal, vol. 83, n 1, 2009, p. 1-26, http:// papers.ssrn.com/sol3/papers.cfm?abstract_id $=1157158$.

Surowiecki, James, « House of cards », in The New Yorker, 16 mars 2009. Disponible en ligne : http://www.newyorker.com/talk/financial/2009/03/16/090316ta_talk_surowiecki.

Traub, Amy, et Catherine Ruetschlin, « The Plastic Safety Net 2012 : National Survey on Credit Card Debt of Low and Middle Income Households ", in Dēmos Report, 22 mai 2012. Disponible en ligne : http://www.demos.org/publication/plastic-safety-net.

United States Government Accountability Office (GAO), Credit Cards : Increased Complexity in Rates and Fees Heightens Need for More Effective Disclosures to Consumers, 11 octobre 2006. Disponible en ligne : http://www.gao.gov/new.items/d06929.pdf. 
Zeldin, Cindy, et Mark Rukavina, « Borrowing to Stay Healthy : How Credit Card Debt is Related to Medical Expenses », in Dēmos Report , Janvier 2007. Disponible en ligne : http://www.aecf.org/ upload/publicationfiles/fes3622h1313.pdf.

\section{NOTES}

1. «Payez votre Visa avec votre MasterCard », traduction de l'auteur.

2. «Unquestionably, innovation and deregulation have vastly expanded credit availability to virtually all income classes. Access to credit has enabled families to purchase homes, deal with emergencies, and obtain goods and services ", traduction de l'auteur extraite du texte original (Greenspan, 2005).

3. "These guys have figured out the best way to compete is to put a smiley face in your commercials, a low introductory rate, and hire a team of MBAs to lay traps in the fine print ", traduction de l'auteur extraite du texte original (Frontline \&NYT, 2004).

4. Voir CreditCards.com (2007) et National Foundation for Credit Counseling (2009)

5. "There are some things that money can't buy. For everything else, there's MasterCard", traduction de l'auteur.

6. "You've put a lot of work into your home. Isn't it time for your home to return the favor?", traduction de l'auteur.

7. « Ni revenus, ni emploi, ni patrimoine », traduction de l'auteur.

8. «(...) This equilibrium between 'good' debtors who are rewarded with free interest as convenience users and 'bad' debtors who are punished with high interest rates as revolvers is increasingly being publicly challenged as morally bankrupt ", traduction de l'auteur extraite du texte original (Manning, $2000: 296-297$ ).

\section{RÉSUMÉS}

La déréglementation du système bancaire aux États-Unis dans les années 1980 s'est accompagnée d'une démocratisation du crédit, mais également d'un marketing de plus en plus agressif de la part des organismes proposant des cartes de crédit revolving. Pendant les années fastes, les Américains se sont laissés séduire par l'argent facile et par l'offre toujours plus tentante de souscription à des cartes qui sont devenues de plus en plus indispensables.

Après l'éclatement de la bulle immobilière et l'effondrement du marché des crédits hypothécaires, le secteur du crédit à la consommation, à son tour, a été touché de plein fouet. Les consommateurs ont alors eu recours à la fuite en avant, contractant de nouveaux emprunts pour financer les précédents, stratégie surnommée ironiquement "paying your Visa with your MasterCard ». Les ménages les plus fragiles ont fait les frais de cet emballement du système : en 2009, les Américains devaient plus de 1000 milliards de dollars aux organismes de cartes de crédit tandis que plus d'1,6 million, surendettés, se déclaraient en faillite personnelle.

Cet article s'attachera à mettre en lumière le faisceau de causes multiples et entrecroisées qui ont jalonné cette chronique d'une catastrophe annoncée.

By focusing on the most glaring abuses of the credit card market prior to the 2010 Dodd-Frank reform, this article analyses the combination of factors which paved the way for the 2008 crisis 
and further widened inequalities between rich and poor Americans.

We start by examining the historical reasons for the boom in the credit card sector, due essentially to the financial deregulation of the 1980s which democratized credit and made credit cards available to less creditworthy customers. Eager to tap these new markets, especially lowerincome groups and students, the financial sector devised aggressive marketing campaigns, and ensnared many customers into a cycle of lifelong debt dependency. Obscure and misleading contracts trapped many Americans into believing they could afford these products, while their ignorance of the complexity of credit markets and their willingness to fall for such promising offers did the rest. This situation gave birth to increasingly risky practices, for example, «paying your Visa with your MasterCard» or taking up second mortgages on their homes, and an increasing number of American households became overindebted, while the level of personal bankruptcy went through the roof : in 2009, over 1,6 million Americans filed for bankruptcy, and they owed over 1000 billion dollars to credit card institutions.

La desregulación del sistema bancario en los Estados Unidos en los años 80 vino acompañada de una democratización del crédito así como de un marketing muy agresivo por parte de los organismos que gestionan tarjetas de crédito revolving. Durante los años fastos, los norteamericanos se dejaron seducir por el dinero fácil y por la oferta cada vez más seductora para suscribir tarjetas que terminaron tornándose indispensables.

Trás el estallido de la burbuja inmobiliaria y el desmoronamiento del mercado de los créditos hipotecarios, le tocó al sector del crédito al consumo sufrir los embistes de la crisis. Los consumidores optaron por la huida hacia adelante, contrayendo nuevos préstamos para financiar los antiguos, estrategia que la frase «Paga tu Visa con tu MasterCard» resume con ironía. Los hogares más frágiles terminaron pagando las consecuencias de esta aceleración del sistema : en el año 2009, los norteamericanos debían más de 1000 billones de dólares a los organismos de tarjetas de crédito y más de un millón y medio, excesivamente endeudados, se declaraban en quiebra.

Este artículo procura analizar las causas múltiples y entrecruzadas que han marcado la crónica de una catástrofe anunciada.

\section{INDEX}

Mots-clés : carte de crédit, déréglementation bancaire, Dodd-Frank, surendettement, score FICO, faillite, crédit renouvelable

Keywords : credit card, banking deregulation, Dodd-Frank, overindebtedness, FICO score, bankruptcy, credit revolving

Palabras claves : tarjeta de crédito, desregulación bancaria, Dodd-Frank, endeudamiento excesivo, FICO Score, quiebra, crédito revolving

\section{AUTEUR}

\section{MARIE-CHRISTINE PAUWELS}

Ancienne élève de l'École Normale Supérieure, Marie-Christine Pauwels est maître de conférences à l'Université de Paris Ouest Nanterre la Défense, où elle enseigne la civilisation américaine. Elle est l'auteur de plusieurs ouvrages sur les États-Unis et a publié de nombreux articles portant sur les questions d'identité et d'intégration des minorités ethniques. Elle est membre du groupe de recherches Politiques Américaines (CREA-Université de Paris OuestNanterre la Défense) et du CERVEPAS (Paris III Sorbonne-Nouvelle). Ses dernières 
publications : « Does Affirmative Action have a Future in Barack Obama's America? », Journal of Intercultural Studies, vol. 32, $\mathrm{n}^{\circ} 3,2011$, Disponible en ligne : http://www.tandfonline.com/doi/ full/10.1080/07256868.2011.565740\#.UjMZZdIcA8k ; « The Credit Card Market in the United States : a Predictable Collapse », in Valérie Peyronel, Catherine Coron et Régine Hollander (dir.), La Crise financière et les économies du monde anglophone, Presses de la Sorbonne Nouvelle, 2010 ; "L'évolution de l'affirmative action aux États-Unis sous la présidence de George W. Bush », article paru dans la revue LISA, vol. 3, $\mathrm{n}^{\circ}$ 1, 2010 : The Legacy of the Bush years : An Economic and Social Assessment, p. 105-123. Disponible en ligne : http://lisa.revues.org/index3385.html ; « La lutte contre les inégalités dans l'enseignement aux États-Unis : premier bilan des 'percentage plans' ", in Christine Zumello et Polymnia Zagefka (dir.), Égalité - Inégalité(s) dans les Amériques, Actes du colloque de l'IHEAL (Institut des Hautes Etudes sur l'Amérique Latine), janvier 2009. mcpauwels1atgmail.com 